\title{
Reaktive Angioendotheliomatose nach Implantation einer Schultergelenksprothese
}

\author{
Reactive Angioendotheliomatosis After Implantation of a Shoulder Joint \\ Prosthesis
}

Autor

Institut

\section{G. Wagner}

Hautklinik, Klinikum Bremerhaven Reinkenheide (Chefarzt: Dr. G. Wagner)

\section{Bibliografie}

DOI 10.1055/s-2006-945091

Akt Dermatol 2007; 33;

42-46 ๑ Georg Thieme

Verlag KG Stuttgart · New York ISSN 0340-2541

Korrespondenzadresse

Dr. Gunnar Wagner

Hautklinik · Klinikum

Bremerhaven Reinkenheide

Postbrookstr. 103 .

27574 Bremerhaven

\section{Zusammenfassung \\ $\nabla$}

Bei einer 72-jährigen Patientin entwickelte sich 6 Wochen nach Implantation einer Schultergelenksprothese ein persistierendes Erythem im Bereich der postoperativen Narbe. Auf der Grundlage histologischer und immunhistologischer Befunde konnte die Diagnose einer reaktiven Angioendotheliomatose gestellt werden. Die

\section{Einleitung}

$\nabla$

Unter der Diagnose einer Angioendotheliomatose werden zwei unterschiedliche Erkrankungen zusammengefasst, deren gemeinsame Ursache in einer intravasalen Tumorzellproliferation zu sehen ist. Neben einer reaktiven oder benignen Form, über die erstmals 1958 von Gottron und Nikolowski berichtet wurde, beschrieben Pfleger und Tappeiner 1959 und 1963 eine maligne Variante, die sie auch als Angioendotheliomatosis proliferans systematisata bezeichneten [1-3]. Besonders die von Pfleger und Tappeiner im „Hautarzt“ publizierten Arbeiten sind auch heute noch außerordentlich interessant und lesenswert, verdeutlichen sie doch die erheblichen Schwierigkeiten, einen bis dahin klinisch und histopathologisch nicht beschriebenen dermatologischen Befund einzuordnen, der darüber hinaus in seiner reaktiven Form als eigenständige Dermatose oder in der malignen Variante als dermatologische Teilmanifestation einer neoplastischen Systemerkrankung verstanden werden kann. Die reaktive Angioendotheliomatose, deren intravasale Zellproliferationen endothelialen Ursprungs sind, gilt als eine sehr seltene, biologisch gutartige und in der Regel selbstlimitierend verlaufende Dermatose. Bei einem Teil der Patienten lassen sich assoziierte Erkrankungen, meist infektiöser Genese, nachweisen. Die maligne Form der Angioendotheliomatose wird heute hingegen reaktive Angioendotheliomatose ist mit verschiedenen, häufig infektiös verursachten Erkrankungen assoziiert. Berichte über eine postoperative Manifestation der reaktiven Angioendotheliomatose fanden sich in der uns zur Verfügung stehenden Literatur nicht. Ob bei der Patientin ein kausaler Zusammenhang zwischen der Operation und dem Auftreten der Dermatose bestand, lässt sich letztendlich nicht sicher entscheiden.

als angiotropes Lymphom definiert, das sich als maligne Systemerkrankung bevorzugt sowohl an der Haut als auch am zentralen Nervensystem manifestiert und trotz Behandlung eine sehr schlechte Prognose aufweist. Das dermatologische Bild der Angioendotheliomatosen ist uncharakteristisch und umfasst ein breites Spektrum verschiedenartiger Befunde, zu denen Erytheme, Knoten, Blasen und Ulzerationen gezählt werden. Die klinische Morphologie erlaubt jedoch keine Unterscheidung zwischen der reaktiven und der malignen Angioendotheliomatose, da die Befunde bei beiden Formen weitgehend identisch sind. Eine vergleichbare Situation besteht auch bei der Beurteilung der histopathologischen Befunde der Angioendotheliomatosen. Dabei lassen sich die richtungsweisenden intravasalen Zellproliferationen, die je nach vorliegender Form aus Endothel- oder aus Lymphomzellen bestehen, häufig nicht sicher differenzieren, so dass die Diagnose einer reaktiven oder einer malignen Angioendotheliomatose bisweilen erst anhand immunhistologischer Befunde gestellt werden kann.

\section{Kasuistik \\ $\nabla$}

\section{Anamnese}

Im Zusammenhang mit einer Rotatorenmanschettenverletzung im rechten Schultergelenk wurde bei der hier vorgestellten 72-jährigen Pa- 


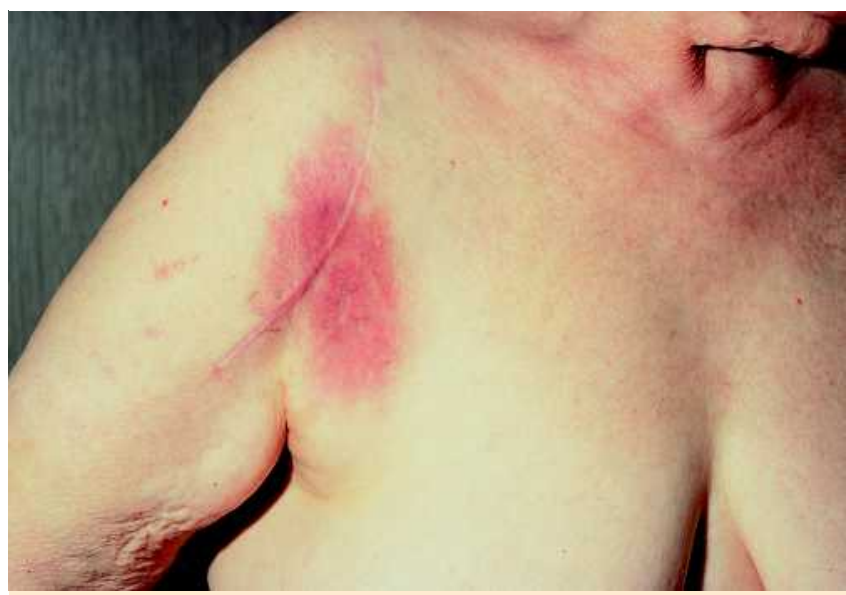

Abb. 1 Unscharf begrenztes Erythem im Bereich der OP-Narbe bei Z. n. Implantation einer Schultergelenksprothese.

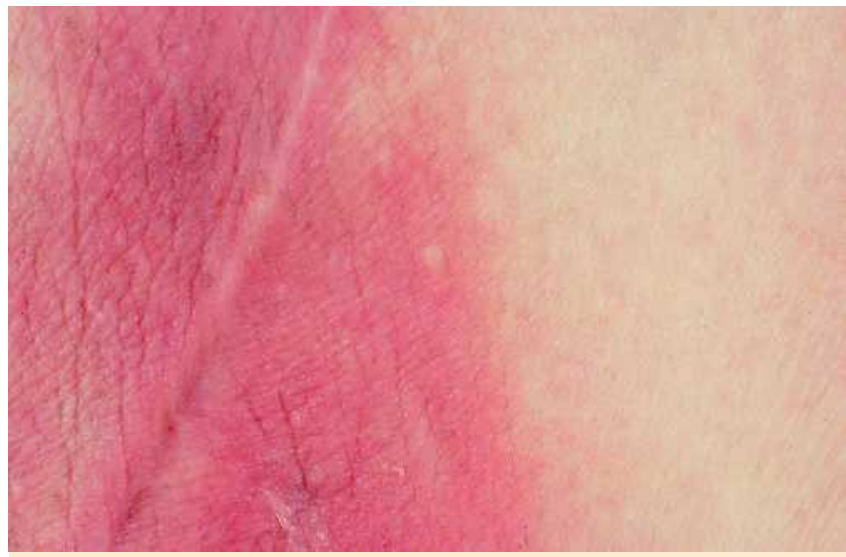

Abb. 2 Detailaufnahme: Monomorphes Erythem mit vergröbertem Hautfaltenrelief.

tientin die Implantation einer Aequalis-Prothese notwendig. Der postoperative Verlauf zeigte zunächst keine Besonderheiten. Nachdem das Schultergelenk 3 Wochen mit einem Gilchrist-Verband versorgt worden war, erfolgte anschließend eine krankengymnastische Behandlung. Dabei wurden erstmals 6 Wochen nach der Operation Hautveränderungen im Narbenbereich beobachtet. Unter der Diagnose einer postoperativen Lymphangitis verordnete der Hausarzt der Patientin eine orale Penicillin-Therapie, die jedoch zu keiner Rückbildung des dermatologischen Befundes führte. Daraufhin wurde die Patientin 2 Monate nach der primären Manifestation der Dermatose in unserer Klinik vorgestellt. In der weiteren Anamnese ergab sich kein Hinweis für eine Atopie oder früher aufgetretene chronisch-entzündliche Dermatosen. Ein bekannter Hypertonus wurde seit 5 Jahren mit Ramipril behandelt. Die Einnahme weiterer Medikamente verneinte die Patientin.

\section{Dermatologischer Befund}

Ventral an der rechten Schulter im mittleren Abschnitt der postoperativen Narbe längsovales, etwa $12 \times 6 \mathrm{~cm}$ großes, ungleichmäßig aufgebautes und unscharf begrenztes Erythem mit umschrieben deutlicher Betonung des Hautfaltenreliefs ( $\bullet$ Abb. 1 u. 2).

\section{Histopathologischer Befund}

In zahlreichen Stufenschnitten weitgehend unauffällige Epidermis. In der ödematös aufgelockerten Dermis Lymphangiektasien und Proliferation von kleinen Blutgefäßen, ausgekleidet von plumpen Endothelzellen ( $\bullet$ Abb. 3 u. $\bullet$ 4).

\section{Immunhistochemischer Zelldifferenzierungsbefund} CD20 negativ, UCHL1 positiv. Myeloperoxidase schwach positiv. Aktin positiv, CD31 positiv ( $\bullet$ Abb. 5). Diagnose: Benigne reaktive Angioendotheliomatose ohne Hinweis für ein kutanes Lymphom oder Angiosarkom (Dr. C. Diaz, Einsendungslabor für Dermatopathologie, Freiburg).

\section{Allergologische Testungen}

Die durchgeführten Epikutantestungen erfolgten nach den Leitlinien der DDG [4]. Dabei wurden folgende Kontaktallergene getestet, ohne dass eine epidermale Sensibilisierung nachgewiesen werden konnte:

- Kaliumdichromat 0,5\%, Kobalt (II)-chlorid 1\%, Nickel (II)-sulfat 5\%, Mangan (II)-chlorid 0,5\%,

- Molybdän (V)-chlorid 2\%, Titan (IV)-oxid 0,1\%, Vanadium-pentoxid 10\%, Benzoylperoxid 1\%,

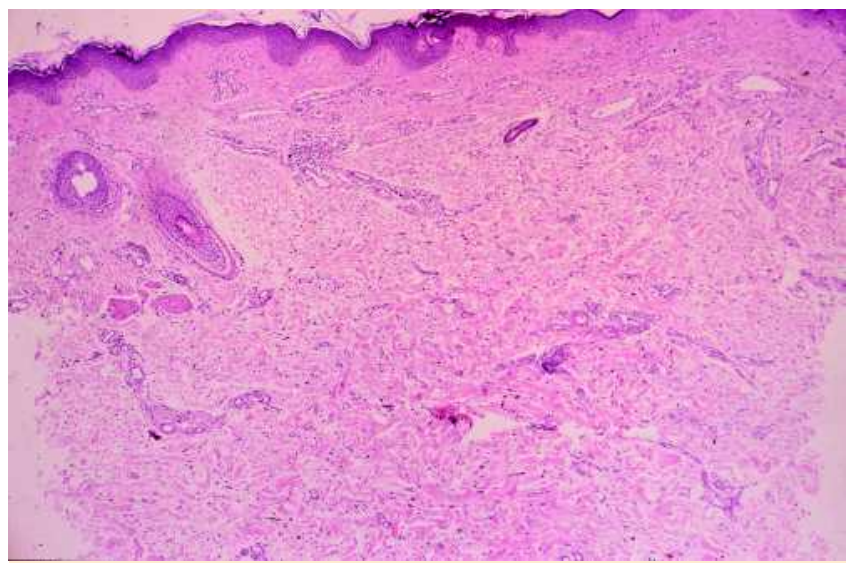

Abb. 3 Diffuse Proliferation von kapillarähnlichen Blutgefäßen in der gesamten Dermis. Die Dermis ist ödematös aufgelockert und zeigt ein diskretes perivaskuläres lympho-histiozytäres Infiltrat (HE × 25).

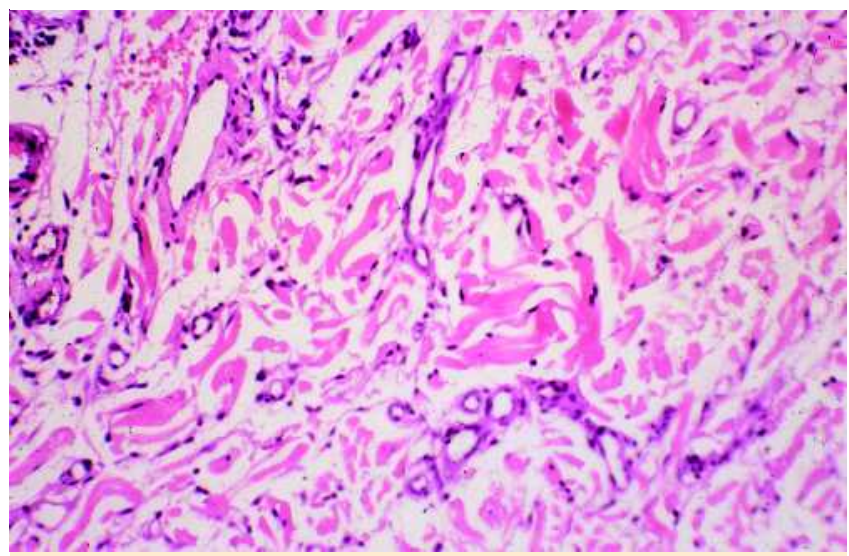

Abb. 4 Zahlreiche auseinanderliegende kleine Blutgefäße mit abgerundeten Luminia zwischen den Kollagenbündeln der ödematös aufgelockerten Dermis $(\mathrm{HE} \times 200)$. 


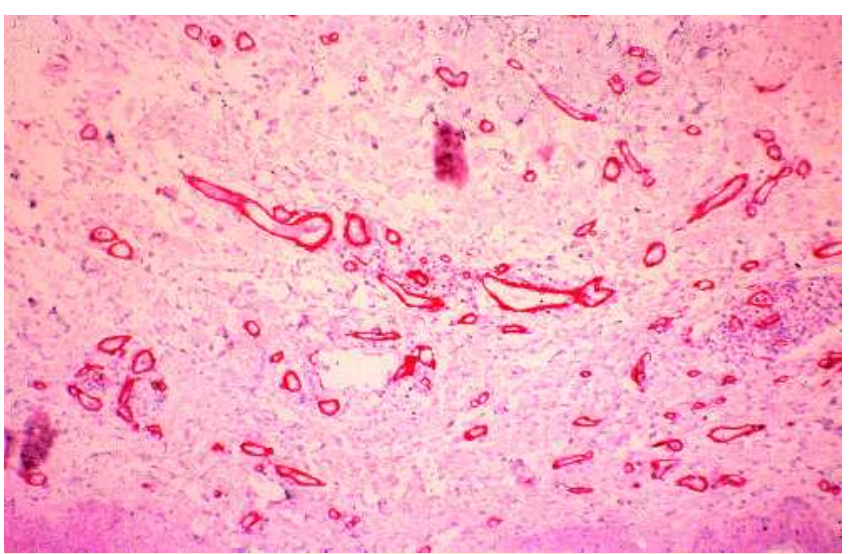

Abb.5 Immunohistochemie: Proliferierende Blutgefäße in der Dermis mit dem Endothelzellmarker CD31 markiert (× 100).

- Gentamycinsulfat 20\%, Hydrochinon 1\%, (2-Hydroxyethyl)methacrylat $1 \%$,

- Methylmethacrylat 1\%, N,N-Dimethyl-P-Toluidin $1 \%$ und Zirkon-dioxid $1 \%$.

\section{Laborbefunde}

Blut- und Differenzialblutbild, Transaminasen, nierenpflichtige Substanzen, Elektrolyte, Immunelektrophorese, Rheumafaktoren, ASL und Urinstatus ohne pathologische Befunde. Hepatitisund Borrelienserologie, antinukleäre Faktoren, Kryoglobuline und Kälteagglutinine ebenfalls unauffällig. In den Stuhluntersuchungen kein Nachweis pathogener Keime oder einer Infestation. Entzündungsparameter: BSG n. W. 90/98 mm, bei Kontrolle $88 / 106 \mathrm{~mm}$ (NW: $10 / 20 \mathrm{~mm}$ ), CRP $63,7 \mathrm{mg} / \mathrm{l}$, bei Kontrolle 63,4 mg/l (NW: 3-15 mg/l). Eiweißelektrophorese geringfügige Erhöhung der alpha-1- und alpha-2-Globulinfraktionen bei sonst unauffälligen Befunden.

\section{Ergänzende Untersuchungen}

Rö.-Thorax, -NNH und -Orthopantomogramm, Mammografie, Lymphknotensonografie zervikal, axillär und inguinal sowie EKG und Echokardiographie ohne richtungsweisende pathologische Befunde. Oberbauchsonographie: mehrere große Konkremente im Gallenblasenkorpus, sonst unauffällige Gallenblase. Keine weiteren pathologischen Befunde im Abdomen. Bei den Konsiliaruntersuchungen der Fachgebiete HNO, MKG, Gynäkologie, Neurologie und Kardiologie keine Hinweise für ein entzündliches Fokalgeschehen oder eine Tumorerkrankung.

Chirurgisches Konsil: Funktion des rechten Schultergelenkes mit einer Flexion bis 90 und einer Abduktion bis 80 Grad ohne wesentliche Einschränkung. Röntgenkontrolle unauffällig, die Prothese ist nicht luxiert. Klinisch und radiologisch kein Anhalt für eine Gelenkinfektion.

\section{Therapie und Verlauf}

Aufgrund der Anamnese, der vorliegenden klinischen Morphologie und der laborchemischen Vorbefunde, die eine deutlich beschleunigte BSG und ein erhöhtes CRP auswiesen, wurde der Befund der Patientin auch unsererseits zunächst als postoperative Lymphangitis eingeordnet. Überraschenderweise zeigte eine orale Behandlung mit $3 \times 500 \mathrm{mg}$ Cefaclor täglich über einen Zeitraum von 3 Wochen keinen Einfluss auf die Ausprägung des klinischen Befundes. Um die nun differenzialdiagnostisch ver- mutete Kontaktdermatitis zu belegen, erfolgte eine Biopsie, deren histopathologische Beurteilung zur Diagnose einer reaktiven Angioendotheliomatose führte. Bei der nachfolgenden umfangreichen Diagnostik konnten die in der Literatur beschriebenen assoziierten Erkrankungen bei unserer Patientin nicht nachgewiesen werden. Allerdings fand sich auch kein klinisches Korrelat der deutlich erhöhten laborchemischen Entzündungsparameter.

Eine probeweise durchgeführte lokale Behandlung mit Prednicarbat blieb ebenfalls wirkungslos. Bei einer Wiedervorstellung der Patientin 6 Monate nach Auftreten der Dermatose fand sich ein insgesamt aufgelockertes und deutlich pigmentiertes Erythem, ohne dass jedoch eine vollständige Rückbildung des Befundes festgestellt werden konnte.

\section{Diskussion \\ $\nabla$}

Die beiden Formen der Angioendotheliomatose, die maligne und die reaktive oder benigne Variante, werden nach heutiger Auffassung als zwei eigenständige Entitäten angesehen, die allerdings identische klinische, nicht voneinander abgrenzbare dermatologische Befunde aufweisen. Die maligne Angioendotheliomatose ist die häufigere der beiden Formen. Domizio et al. berichteten 1989 über 101 publizierte Fälle der malignen Form, während Kutzner et al. 1991 die Zahl der bekannt gewordenen reaktiven Angioendotheliomatosen mit weniger als 10 Erkrankungen angaben [5,6]. Bei späteren Untersuchungen von Lazova et al. und McMenamin und Fletcher fanden sich nur wenige neue Erkrankungsfälle. Den Autoren zufolge waren bis 199617 Fälle und bis 200235 Fälle der reaktiven Form publiziert worden $[7,8]$. Die klinische Morphologie der Angioendotheliomatosen ist vielfältig und kann bei den einzelnen Patienten sehr unterschiedliche, teilweise monomorphe, teilweise polymorphe Befunde aufweisen. Beim Auftreten einzelner Effloreszenzen zeigen sich diese bevorzugt an den Unterschenkeln und überraschenderweise auch immer wieder im Bereich der Ohrmuscheln, während bei einer generalisierten Ausbreitung sowohl der Stamm als auch die oberen und unteren Extremitäten bevorzugt betroffen sind [9-12]. Am häufigsten finden sich kleinmakulöse oder flächenhafte Erytheme in unterschiedlicher Ausprägung. Neben hellroten, braunroten oder lividen Farbtönen werden urtikarielle, derb infiltrierte, retikuläre oder purpuriforme Erytheme beschrieben. Die unscharf begrenzten Erytheme können durch zahlreiche Telangiektasien gekennzeichnet sein und werden von einzelnen Patienten ungewöhnlicherweise als druckschmerzhaft empfunden [11 - 14].

Eine weitere klinische Manifestation der Angioendotheliomatosen sind kutan oder subkutan lokalisierte Knoten und Plaques, die sich ebenfalls durch rote oder livide Farbtöne auszeichnen und eine derbe Konsistenz aufweisen $[6,11,15]$. Darüber hinaus werden vereinzelt auch Blasen beschrieben $[9,12]$. In der Regel treten sowohl die nodulären als auch die bullösen Effloreszenzen in Kombination mit den verschiedenen Erythemformen auf. Alle primären Effloreszenzen der Angioendotheliomatosen können nekrotisieren und im Verlauf ihrer weiteren Entwicklung hämorrhagische Krusten, Erosionen und Ulzera ausbilden $[11,12]$. Im Gegensatz zur reaktiven Angioendotheliomatose, deren klinische Symptomatik auf das Hautorgan beschränkt bleibt, zeigt die maligne Form als angiotropes Lymphom eine systemische Ausbreitung mit deutlich bevorzugter neurokutaner Manifestation. Die neurologische Symptomatik der malignen Angio- 
endotheliomatose ist dabei häufiger als die kutane Beteiligung und zeigt sich unter anderem in Form von transitorischen ischämischen Attacken, Krampfanfällen, Parästhesien, Ataxien, Gleichgewichtsstörungen, Wesensveränderungen und Demenz [16 - 18]. Dem biologischen Verhalten einer malignen Systemerkrankung folgend können zahlreiche weitere Organe betroffen sein. Neben dem Gastrointestinal- und dem Urogenitaltrakt finden sich in der Literatur Berichte über spezifische Infiltrationen der Lungen, der Leber und der Nieren sowie weitere Manifestationen im Bereich der Knochen, der Lymphknoten und der Milz [19]. Das histopathologische Bild der Angioendotheliomatosen ist durch intravasale Tumorzellproliferationen gekennzeichnet, die in kleineren und mittelgroßen Gefäßen des Koriums und der Subkutis nachweisbar sind. Je nach Ausmaß der intravasalen Proliferationen können die Gefäßlumina durch fibrinoide Thromben partiell oder vollständig verschlossen sein $[8,29]$. Bei der malignen Angioendotheliomatose bestehen die Proliferate aus atypischen mononukleären Zellen mit zahlreich vorkommenden Zell- und Kernatypien, die in den meisten der bisher publizierten Fälle als B-Lymphozyten klassifiziert werden konnten [6,21]. Neben diesen intravasalen oder angiotropen B-ZellLymphomen wurden nur sehr vereinzelt T-Zell-Lymphome oder histiozytische Lymphome diagnostiziert $[11,22,23]$.

Bei der reaktiven Form der Angioendotheliomatose finden sich als charakteristische Befunde intravasale zellreiche Endothelzellproliferationen und extravasal lokalisierte Perizytenmanschetten, während Kernatypien nur sehr vereinzelt beobachtet werden können [8]. In Zweifelsfällen sind für die differenzialdiagnostische Abgrenzung zwischen der malignen und der reaktiven Angioendotheliomatose immunhistologische Untersuchungen notwendig. Der Panleukozytenmarker Anti-LCA (CD45) sowie Antikörper gegen B- und T-Zell-assoziierte Antigene sichern bei positiven Befunden die Diagnose einer malignen Angioendotheliomatose und erlauben darüber hinaus eine weitere Subtypisierung des vorliegenden Lymphoms [24]. Positive Markierungen der intravasalen Proliferationen mit Endothelzellmarkern, z.B. mit UEA1, sowie der Nachweis muskelaktin-besetzter perivasaler Perizytenmanschetten belegen hingegen das Vorliegen einer reaktiven Angioendotheliomatose [21]. Die Ätiopathogenese der Angioendotheliomatosen ist weitgehend ungeklärt. Bei der malignen Form werden als Ursache der Angiotropie der Lymphome spezifische Adhäsionsmoleküle vermutet, die eine Bindung zwischen den Endothel- und Lymphomzellen vermitteln könnten [25]. Einer anderen Hypothese zufolge wird die intravasale Proliferation möglicherweise durch defekte Rezeptoren der Lymphomzellen verursacht [26].

Die Diskussion über die Ätiologie und die Pathogenese der reaktiven Angioendotheliomatose ist geprägt durch das gleichzeitige Auftreten assoziierter Erkrankungen. Hierzu zählen vorrangig bakterielle Infektionen wie die auffällig häufig beobachtete subakute bakterielle Endokarditis, aber auch die Tuberkulose oder die Otitis media [7,12]. Darüber hinaus finden sich in der Literatur einzelne Berichte über assoziierte Gefäßprozesse, z.B. eine portale Hypertension bei alkohol-toxischer Zirrhose oder bei arteriovenösen Shunts [27,28]. Bei einer weiteren Patientengruppe mit reaktiver Angioendotheliomatose konnten Kryoglobuline, Kälteagglutinine und Antiphospholipid-Antikörper als pathologische Serumbestandteile nachgewiesen werden [29-31]. Allen assoziierten Erkrankungen gemeinsam sind pathogenetische Konzepte, die die intravasale Endothelzellproliferation als Folge einer gestörten Durchblutung der betroffenen Hautareale beschreiben. Neben infektallergischen Mechanismen, z. B. im Sinne einer Immunkomplex-Vaskulitis, wird auch die Möglichkeit einer verstärkten Produktion von Angiogenesefaktoren als Folge einer intravasalen Thrombenbildung und der damit verbundenen Hypoxie diskutiert $[8,32,33]$. Der Nachweis assoziierter Erkrankungen ist bei der reaktiven Angioendotheliomatose jedoch keineswegs obligat. Bei einem Teil der Patienten fanden sich weder Begleiterkrankungen noch pathologische Laborbefunde [7]. Auch bei der hier vorgestellten Patientin konnten die bisher beschriebenen assoziierten Erkrankungen nicht nachgewiesen werden. Die reaktive Angioendotheliomatose der Patientin war 6 Wochen nach Implantation einer Schultergelenksprothese im Narbenbereich aufgetreten. Der Zeitraum und die Lokalisation sprechen zunächst für einen kausalen Zusammenhang zwischen dem Auftreten der Dermatose und dem operativen Eingriff. Unterstellt man die Möglichkeit einer operativ verursachten Hypoxie, z. B. durch arterielle Gefäßligaturen, ließe sich das postoperative Auftreten der Dermatose auch pathogenetisch erklären. Der operative Eingriff und die anschließende Wundheilung waren bei unserer Patientin jedoch komplikationslos verlaufen, so dass eine Ischämie und eine damit verbundene Hypoxie als Ursache der reaktiven Angioendotheliomatose im vorliegenden Fall unwahrscheinlich sind. Darüber hinaus müsste man bei einer traumatischen Genese der reaktiven Angioendotheliomatose und der Anzahl der täglich durchgeführten Operationen wohl häufiger mit dem Auftreten der Dermatose rechnen, als dies tatsächlich der Fall ist. So fand sich in der uns zugänglichen Literatur kein weiterer Fall einer postoperativ aufgetretenen reaktiven Angioendotheliomatose. Unabhängig von der postoperativen Manifestation der reaktiven Angioendotheliomatose bleibt bei unserer Patientin auch die pathogenetische Bedeutung der erhöhten laborchemischen Entzündungsparameter unklar, zumal deren Ursache ebenfalls nicht aufgedeckt werden konnte. Dabei fanden sich keine Hinweise für akut oder persistierend infektiöse, myeloproliferative oder autoimmunologische Erkrankungen, so dass eine Ätiologie der reaktiven Angioendotheliomatose in der hier wiedergegebenen Kasuistik trotz des zeitlichen und topographischen Zusammenhanges mit der vorausgegangenen Operation als nicht geklärt angesehen werden muss.

In Abhängigkeit von der jeweils vorliegenden Morphologie der Angioendotheliomatose müssen klinisch zahlreiche Dermatosen differenzialdiagnostisch berücksichtigt werden, z.B. die Lymphangiosis carcinomatosa viszeraler Neoplasien, bakterielle Lymphangitiden, die Embolia cutis medicamentosa, frühe Formen der Mycosis fungoides, umschriebene Ekzemmanifestationen sowie die verschiedenen Vaskulitiden [7,12,32]. Bei dieser Vielzahl möglicher Differenzialdiagnosen verwundert es nicht, dass die Diagnose einer Angioendotheliomatose in der Regel erst aufgrund histopathologischer Untersuchungen gestellt wird. Die gleiche Einschätzung gilt im Übrigen auch für die diffuse dermale Angiomatose, die von einigen Autoren als Variante der reaktiven Angioendotheliomatose, von anderen hingegen als eigenständige Dermatose verstanden wird [33,34].

Die Behandlung der malignen Angioendotheliomatose entspricht den Therapieempfehlungen hochmaligner Non-Hodgkin-Lymphome. Neben einer kombinierten Chemotherapie, z.B. nach dem CHOP-Schema, werden bei umschriebenen Organmanifestationen zusätzlich radioonkologische Therapieverfahren eingesetzt. Nichtsdestotrotz muss die Prognose mit einer durchschnittlichen Überlebenszeit von nur einem Jahr als außergewöhnlich ungünstig angesehen werden $[12,26,35]$. Bei der reaktiven Angioendotheliomatose sollte zunächst eine Behandlung assoziierter Erkrankungen erfolgen. Darüber hinaus sind über- 
wiegend systemische Behandlungsversuche mit verschiedenen Antibiotika durchgeführt worden. Einzelne Patienten erhielten zusätzlich eine systemische oder topische Therapie mit Glukokortikoiden [7]. Bei allen Behandlungsversuchen sollte jedoch berücksichtigt werden, dass spontane Rückbildungen der reaktiven Angioendotheliomatose keineswegs ungewöhnlich sind $[11,36]$.

\section{Abstract}

\section{Reactive Angioendotheliomatosis After Implantation of a Shoulder Joint Prosthesis}

Six weeks after implantation of a shoulder joint replacement a persistent erythema developed on the site of the scar in a 72 years old female patient. Histologic and immunohistochemical findings revealed diagnosis of reactive angioendotheliomatosis. This disease is associated with different disorders frequently caused by infections. No report about a postsurgical manifestation of a reactive angioendotheliomatosis was found in the literature. However, one cannot conclude from this that manifestation of the described skin disease is causally related to the previous operation.

\section{Literatur}

1 Gottron HA, Nikolowski W. Extrarenale Löhlein-Herdnephritis der Haut bei Endocarditis. Arch Klin Exp Dermatol 1958; 207: 156 - 176

2 Pfleger L, Tappeiner J. Zur Kenntnis der systematisierten Endotheliomatose der kutanen Blutgefäße. Hautarzt 1959; 10: 359- 363

3 Tappeiner J, Pfleger L. Angioendotheliomatosis proliferans systemisata. Hautarzt 1963; 14: 67-70

4 Schnuch A, Aberer W, Agathos M et al. Leitlinien der Deutschen Dermatologischen Gesellschaft (DDG) zur Durchführung des Epikutantests mit Kontaktallergenen. Hautarzt 2001; 52: 864-866

5 Domizio P, Hall PA, Cotter $F$ et al. Angiotropic large cell lymphoma (ALCL). Hematol Oncol 1989; 7: 195-206

6 Kutzner H, Englert W, Hellenbroich $D$ et al. Angioendotheliomatosis proliferans systemisata: eine kutane Manifestation maligner B-Zellen-Lymphome. Hautarzt 1991; 42: 384-390

7 Lazova R, Slater C, Scott G. Reactive Angioendotheliomatosis. Am J Dermatopathol 1996; 18: 63-69

8 McMenamin ME, Fletcher CD. Reactive Angioendotheliomatosis. Am J Surg Pathol 2002; 26: 685-697

9 Schmidt K, Hartig C, Stadler R. Reaktive Angioendotheliomatose bei chronisch lymphatischer Leukämie. Hautarzt 1996; 47: 550-555

10 Kolde G. Angioendotheliomatosis proliferans systematisata. Hautarzt 1991; 42: 589-592

11 Schley G, Kauffold D, Guse E et al. Angioendotheliomatosis proliferans systematisata. Hautarzt 2001; 52: 1030-1034

12 Wallenfang K, Stadler R. Angioendotheliomatose. Akt Dermatol 2002; 28: $374-379$

13 Berger TG, Dawson NA. Angioendotheliomatosis. J Am Acad Dermatol 1988; 18: $407-412$
14 Gupta AK, Lipa M, Haberman HF. Proliferating angioendotheliomatosis. Case with long survival and review of literature. Arch Dermatol 1986; 122: $314-319$

15 Eisert J. Skin manifestations of subacute bacterial endocarditis: Case report of subacute bacterial endocarditis mimicking Tappeiner's angioendotheliomatosis. Cutis 1980; 25: $394-400$

16 Beal MF, Fisher CM. Neoplastic angioendotheliosis. J Neurol Sci 1982; 53: 359- 375

17 Berger TG, Dawson NA. Angioendotheliomatosis. J Am Acad Dermatol 1988; 18: $407-412$

18 Bots GT. Angioendotheliomatosis of the central nervous system. Acta Neuropathol (Berl) 1974; 28: 75 - 78

19 Wick MR, Banks PM, McDonald TJ. Angioendotheliomatosis of the nose with fatal systemic dissemination. Cancer 1981; 48: 2510-2517

20 Cooper PH. Angioendotheliomatosis: two separate diseases. J Cutan Pathol 1988; 15: 259

21 Wick MR, Rocamora A. Reactive and malignant "angioendotheliomatosis": a discriminant clinicopathological study. J Cutan Pathol 1988; 15: 260-271

22 Ansell J, Bhawan J, Cohen S et al. Histiocytic lymphoma and malignant angioendotheliomasis: one disease or two? Cancer 1982; 50: $1506-$ 1512

23 Sepp N, Schuler G, Romani N et al. "Intravascular lymphomatosis" (angioendotheliomatosis): evidence for a T-cell origin in two cases. Hum Pathol 1990; 21: 1051 - 1058

24 Hauschild A, Sterry W. Formalinresistente Leukozyten-Differenzierungsantigene in der Dermatohistopathologie. Hautarzt 1990; 41: $345-352$

25 Springer TA. Adhesion receptors of the immune system. Nature 1990; 346: $425-434$

26 Kusaba T, Hatta T, Tanda $S$ et al. Histological analysis on adhesive molecules of renal intravascular large B cell lymphoma treated with CHOP chemotherapy and rituximab. Clin Nephrol 2006; 65: 222 - 226

27 Quinn TR, Alora MBT, Momtaz KT et al. Reactive angioendotheliomatosis with underlying hepatopathy and hypertensive portal gastropathy. Int J Dermatol 1998; 37: 378 - 385

28 Requena L, Farina MC, Renedo G et al. Intravascular and diffuse dermal reactive angioendotheliomatosis secondary to iatrogenic arteriovenous fistulas. J Cutan Pathol 1999; 26: 159-164

29 Creamer D, Black MM, Calonje E. Reactive angioendeotheliomatosis in association with the antiphospholipid syndrome. J Am Acad Dermatol 2000; 42: $903-906$

30 LeBoit PE, Solmon AR, Santa Cruz DJ et al. Angiomatosis with luminal cryoprotein deposition. J Am Acad Dermatol 1992; 27: 969-973

31 Porras-Luque JI, Fernández-Herrera J, Daudén E et al. Cutaneous necrosis by cold agglutinins associated with glomeruloid reactive angioendotheliomatosis. Br J Dermatol 1998; 139: 1068 - 1072

32 Martin S, Pitcher D, Tschen J et al. Reactive angioendotheliomatosis. J Am Acad Dermatol 1980; 2: 117-123

33 Kimyai-Asadi A, Nousari HC, Ketabchi $N$ et al. Diffuse dermal angiomatosis: a variant of reactive angioendotheliomatosis with artherosclerosis. J Am Acad Dermatol 1999; 40: 257-259

34 Kutzner H, Requena R, Mentzel T et al. Diffuse dermale Angiomatose. Hautarzt 2002; 53: 808-812

35 Reiner M, Marschall FM, Beck P. Intravaskuläre Lymphomatose (angiotropes Lymphom). Dtsch med Wschr 1998; 123: 1303-1307

36 Rongioletti F, Rebora A. Cutaneous reactive angiomatoses: Patterns and classification of reactive vascular proliferation. J Am Acad Dermatol 2003; 49: 887-896 